\title{
Quantum interference in atomic-sized point contacts
}

\author{
C. Untiedt, ${ }^{*}$ G. Rubio Bollinger, S. Vieira, and N. Agrait \\ Laboratorio de Bajas Temperaturas, Departamento de Física de la Materia Condensada C-III, Universidad Autónoma de Madrid, \\ E-28049 Madrid, Spain \\ and Instituto Universitario de Ciencia de Materiales "Nicolás Cabrera," Facultad de Ciencias, Campus de Cantoblanco, \\ 28049 Madrid, Spain \\ (Received 14 March 2000)
}

\begin{abstract}
The conductance of atomic-sized metallic point contacts is shown to be strongly voltage dependent due to quantum interference with impurities even in samples with low impurity concentrations. Transmission through these small contacts depends not only on the local atomic structure at the contact but also on the distribution of impurities or defects within a coherence length of the contact. In contrast with other mesoscopic systems we show that transport through atomic contacts is coherent even at room temperature. The use of a scanning tunneling microscope (STM) makes it possible to fabricate one atom contacts of gold whose transmission can be controlled by manipulation of the contact allowing inelastic spectroscopy in such small contacts.
\end{abstract}

The conductance of atomic-sized contacts has been extensively studied in relation with the question of conductance quantization. Experimentally, the contacts are fabricated stretching a metallic contact using a scanning tunneling microscope ${ }^{1}$ (STM) or a mechanically controlled break junction (MCBJ). ${ }^{2}$ Theoretically, the point contact has been modeled as a constriction for free electrons,${ }^{3,4}$ or a tight-binding model using different atomic arrangements. ${ }^{5,6}$

Electronic transport in these nanoscopic size structures is coherent, and the conductance at zero bias voltage is given by the Landauer formula ${ }^{7}$

$$
G=G_{0} \sum_{n=1}^{N} T_{n}
$$

where $G_{0}=2 e^{2} / h$ is the conductance quantum ( $e$ is the electron's charge and $h$ is Planck's constant), $N$ is the number of channels in the contact, and $T_{n}$ is the transmission probability of the $n$th channel. It has been recently shown that the number of channels in a one-atom contact depends on the chemical nature of the element. ${ }^{8}$ Simple metals with a $1 \mathrm{~s}$ electronic structure $(\mathrm{Na}, \mathrm{Au})$ have only one channel, while atoms with more complex electronic structures $(\mathrm{Al}, \mathrm{Pb}, \mathrm{Nb})$ have several channels. The transmissions of these channels vary from zero to one and are affected by the atomic configuration of the atoms at the contact and the state of strain. ${ }^{9}$ In the case of $\mathrm{Au}$, the conductance of a one-atom contact is always very close to $G_{0}$ indicating that its single channel is, typically, almost completely open. The inset of Fig. 1 shows the typical behavior observed when breaking a gold contact. The abrupt changes and plateaus in the conductance correspond to atomic rearrangements and elastic deformation, respectively. ${ }^{10}$ The last contact before breaking is likely to be a one-atom contact ${ }^{1}$ and in the case of Au it will consist of a single conductance channel. ${ }^{8}$

The presence of defects or impurities or wall roughness in the constriction has been shown to cause a decrease in the conductance, ${ }^{4,5}$ however, not much attention has been paid to the variation of conductance with voltage. In the calculations most of the times conductance is computed at zero bias voltage, while in the experiments the conductance is typically extracted from the measured current at a fixed low bias voltage (of the order of millivolts). However, as shown in Fig. 1 the conductance of these small contacts is always voltage dependent. This effect was first reported by Bas Ludolph et al. ${ }^{11}$ they measured the voltage dependence of the conductance $\left(\partial G / \partial V=\partial^{2} I / \partial V^{2}\right)$ statistically averaging over a large number of contacts, and showed that its amplitude is suppressed for conductances near $G_{0}$. Here we have studied how can we understand the conductance oscillation pattern for each contact, which information can we obtain from it,
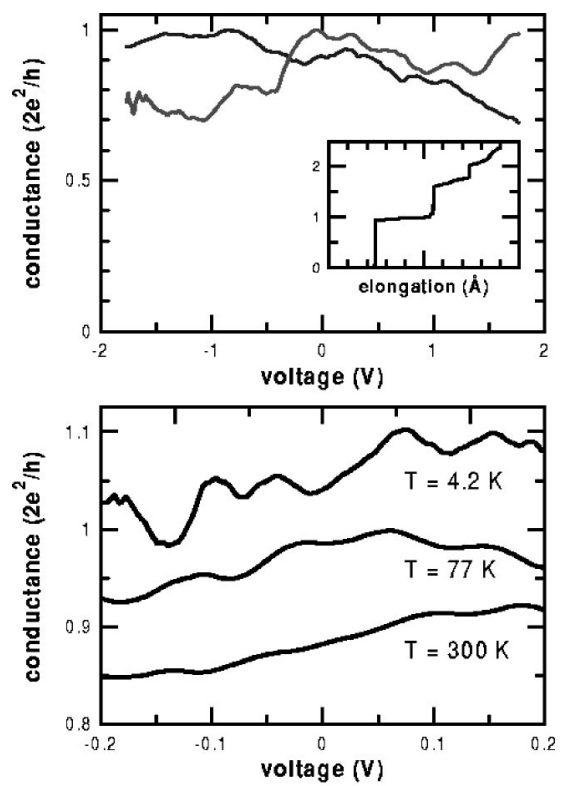

FIG. 1. Conductance vs voltage for different one-atom contacts; (a) two different one-atom contacts at $4.2 \mathrm{~K}$; (b) three different one-atom contacts at $4.2 \mathrm{~K}, 77 \mathrm{~K}$ and $300 \mathrm{~K}$ (the two first are displaced vertically 0.18 and 0.09 units, respectively, for clarity). Inset in (a): the current at fixed voltage during rupture. 
and how can it be modified. In order to obtain this information we have measured the differential conductance as a function of voltage. Using a stable low temperature STM. The samples were high purity $\mathrm{Au}(99.99 \%$ pure), and the experiments where performed at $4.2 \mathrm{~K}$. The differential conductance was measured using a lock-in amplifier, with a modulation of the order of $1 \mathrm{mV}$ and at a frequency of 7 $\mathrm{kHz}$, the typical acquisition time is of several seconds. Figure 1 shows the differential conductance vs voltage of different one-atom gold point-contacts at different temperatures. The conductance is typically asymmetric and presents a complex pattern of oscillations which is stable and reproducible for a given contact but varies greatly for different contact realizations.

The conductance oscillations shown in Fig. 1 are due to quantum interference: the electron wave transmitted through the contact is backscattered to the contact by impurities or defects in the bulk and then partially reflected at the contact, this wave interferes with the directly transmitted wave and modifies the total conductance. ${ }^{11}$ For a constriction with a single transmitted channel, we can easily estimate the amplitude of the interfering wave. Let us assume that there is an impurity of cross section $\sigma$ located at distance $d$ from the constriction, the amplitude reflected into the originally transmitted channel is then $\sim \sqrt{3 \sigma} t_{c} r_{c} / k d^{2}$, where $t_{c}$ and $r_{c}$ are the transmission and reflection coefficients of the constriction, respectively, and $k$ is the electron wave number. The total transmission is given by

$$
T \approx T_{c}\left[1+2 \sqrt{R_{c} R_{1}} \cos \left(2 k d+\phi_{1}\right)\right]
$$

where $\phi_{1}$ depends on the details of the scattering process, $r_{1} \sim a / d^{2} k, R_{c}=r_{c} r_{c}^{*}, T_{c}=t_{c} t_{c}^{*}$, and $R_{1}=r_{1} r_{1}^{*}$. That is for each mode the impurity acts like a transparent barrier with a small reflection coefficient $R_{1} \sim 3 \sigma / d^{4} k^{2}$. The interference term causes the transmission to oscillate as the wave number of the injected electron varies, with a period $e \Delta V$ given approximately by $\sim(2 \pi / k) E_{F} / d$, and an amplitude proportional to $1 / d^{2}$. The current per transmitted mode at zero temperature is given by

$$
I=\frac{2 e}{h} \int_{-e V / 2}^{e V / 2} T(E, V) d E,
$$

where $T$ depends on the energy $E$ and voltage drop $V$ through the wave numbers on each side of the contact, $k_{R}^{2}$ $=2 m / \hbar^{2}\left(E_{F}+E-e V / 2\right), k_{L}^{2}=2 m / \hbar^{2}\left(E_{F}+E+e V / 2\right)$. The conductance can be approximated by $G \approx \frac{1}{2}[T(e V / 2, V)+T$ $(-e V / 2, V)]$. Thus for each voltage the conductance depends on the interference of hot electrons with energy $e V / 2$ above the Fermi level on one side and hot holes with energy $e V / 2$ below the Fermi level on the other side.

Quantum interference effects have been extensively studied in various mesoscopic systems where they show as magnetoconductance fluctuations. In samples with diffuse electron transport (elastic mean free path $l_{e}$ much smaller than sample size), they are called universal conductance fluctuations (UCF) and have the property that at zero temperature their rms. amplitude is of the order of $e^{2} / h$, independent of

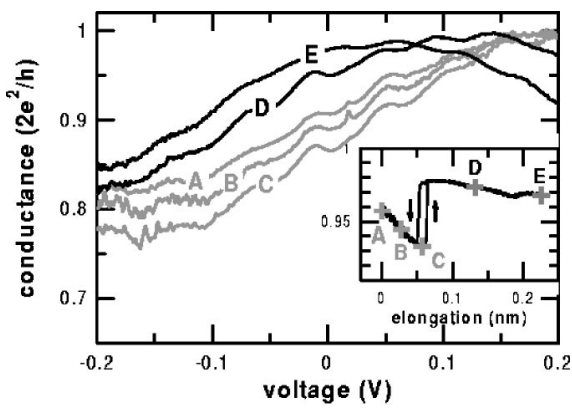

FIG. 2. Experimental changes in the conductance as contact is deformed elastically. Measured at $4.2 \mathrm{~K}$.

sample size and degree of disorder. ${ }^{12}$ The conductance fluctuations as a function of voltage (for voltages up to $2 \mathrm{mV}$ ) have been examined in a diffusive conductor by inserting a tunnel junction. ${ }^{13}$ They have also been observed in ballistic metallic point contacts (of dimensions much larger than atomic size). ${ }^{14}$ Both point contact and tunnel junction serve as injectors of electrons in a specific energy range and electrons with energies different to the Fermi energy can be studied. Very recently, it has been shown by studying statistically many contacts of $\mathrm{Au}$ that in one-atom contacts there is a marked suppression of the interference oscillations when the conductance of the contact is an integer number of $G_{0} .{ }^{11}$

Experimentally, it is observed that slight modifications of the constriction induced by moving the tip a small distance (of the order of $1 \AA$ ) can have a dramatic effect on the interference pattern of the conductance, while the overall value of the conductance remains almost constant (see Fig. 2). These changes are clearly associated with atomic rearrangements in the region of the constriction. Figure 3 illustrates how a very small displacement of a scattering center (defect or local disorder) very close to the constriction is sufficient to cause the observed changes.

We can use this effect to have some control on the atomic arrangement at the contact. A one-atom contact can be manipulated to have a transmission $T_{c}$ very close to one (Fig. $4)$. In this case $R_{c} \approx 0$ and interference effects would be very small. Once the interference is minimized the decrease in conductance due to inelastic scattering by phonon emission which is otherwise hidden by a larger amplitude interference pattern becomes visible (see Fig. 4). The inset of Fig. 4

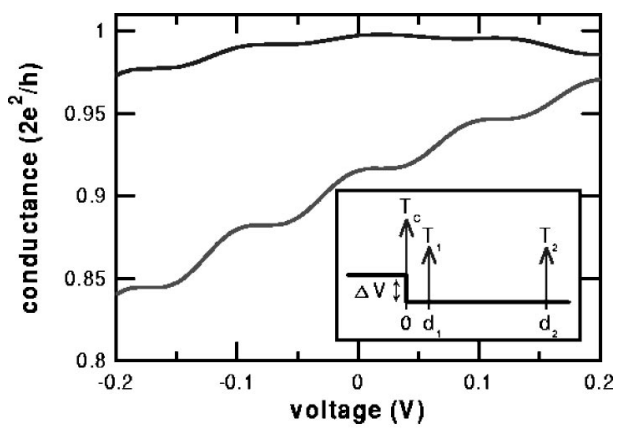

FIG. 3. Calculated conductance for two transparent barriers. Changes in the position of the barrier close to the contact can have a large effect on the conductance. For the black curve $d_{1}=4.3 \AA$, and for the gray curve $d_{1}=4.1 \AA$. The rest of the parameters are identical in both cases $d_{2}=300 \AA, T_{c}=0.8, T_{1}=0.76, R_{2}=10^{-4}$. 


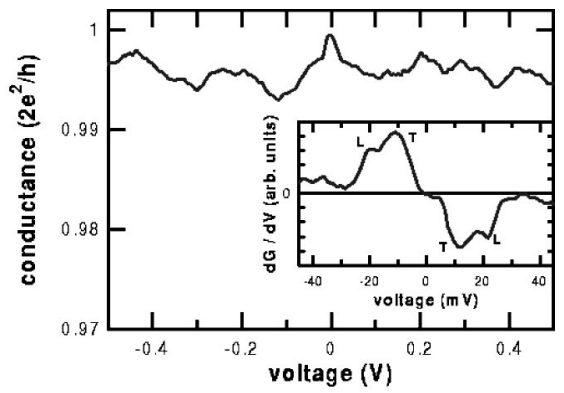

FIG. 4. Experimental conductance. The inset shows the derivative of the conductance were the peaks due to transversal $(T)$ and longitudinal $(L)$ phonons in one-atom contact are seen.

shows the derivative of the conductance which is proportional to the phonon density of states. ${ }^{19}$ The volume probed by the electrons must be of atomic dimensions.

These quantum interference effects are not restricted to high transmission constriction All these results are also valid for low transmission one-atom contacts, that is, in the tunneling regime, which is the conventional operation mode for the STM. In Fig. 5, we show several conductance curves taken at the same location on the sample in the tunneling regime at different tip-to-sample distances (note the logarithmic scale). The relative amplitude of the oscillations is independent of the transmission of the contact, as expected from Eq. (2) for contacts with low transmission. These effects can be relevant for scanning tunneling spectroscopy. ${ }^{15}$ Interference effects due to monoatomic steps ${ }^{16}$ or to adatoms placed on a surface ${ }^{17}$ have been observed in STM experiments at low temperature.

The conductance of one-atom contacts in Fig. 1 oscillations of several periods are clearly discernible indicating contributions of many scattering events. In fact in any event in the case of very pure samples an electron will have the opportunity of seeing many impurities before losing its coherence. Let us estimate the effect of taking into account the contribution to scattering due to many impurities the total transmission can be written as

$$
T \approx T_{c}\left[1+2 \sum_{j} \sqrt{R_{c} R_{j}} \cos \left(2 k d_{j}+\phi_{j}\right)\right]
$$

where $d_{j}$ is the distance from the contact to impurity $j$ and $R_{j} \sim 1 / d^{4}$ as in Eq. (2), and we have only taken into account single scattering processes for simplicity. For a sample with impurity concentration $n$, the number of impurities contributing at any given distance $d$ to the constriction will be proportional to $n d^{2}$, and since all of these impurities contribute to the interference amplitude with the same period but a random phase, the total amplitude corresponding to distance $d$ will be proportional to $\sqrt{n d^{2}}$. Taking into account that the reflection coefficient of the impurities is $\sim 1 / d^{2}$ we obtain a $1 / d$ dependence for the amplitude at a given frequency. A similar reasoning shows that multiple scattering events will also result in the same dependence. Figure 6 shows the amplitude of the components corresponding to different distance for different one-atom contacts, obtained from the maxim of the Fourier spectra (we have made for the $x$ axes

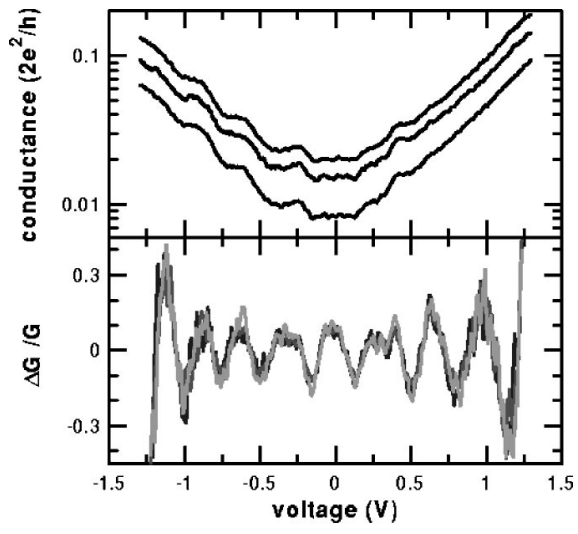

FIG. 5. Conductance oscillations in the tunneling regime. In the lower pannel is also shown the same curves with a low pass filter. Measured at $4.2 \mathrm{~K}$.

the transformation $d=\pi / k$ from the theory above). Contributions of scattering paths of up to $1000 \AA$ are observable. ${ }^{18}$ We can conclude that scattering is caused by a uniform distribution of scattering centers.

In contrast to quantum interference in other mesoscopic systems which typically manifests only at milikelvin temperatures, quantum interference in atomic-sized contacts shows even at room temperature, as shown in Fig. 1(b). The structure of the curves decreases with increasing temperature reflecting the decrease in coherence length. From the discussion in the preceding paragraphs, interference effects will show if there are impurities within a coherence length of the contact, for $\mathrm{Au}$ at room temperature this length is $300 \AA$ (coinciding with the mean free path since at this temperature the main scattering mechanism is electron-phonon collisions) which is larger than the mean distance between impurities for our sample.

The contact plus the impurities within the coherence length constitute a mesoscopic device, whose size decreases with increasing temperature. The transmission $T_{n}$ of each mode in the Landauer formula is voltage dependent and cannot be extracted from a measurement at a fixed bias (or zero). The total transmission of this mesoscopic device can be varied by manipulation of the atomic arrangement at the contact. We have shown that, in the case of $\mathrm{Au}$, this device can have a single conductance channel which can be almost completely open. These quantum interference effects are important in one-atom point-contacts and tunneling atomic junc-

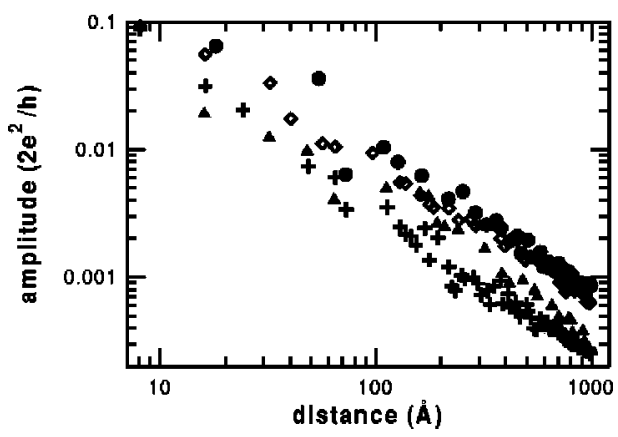

FIG. 6. The frequency dependence of the amplitude for different experimental one-atom contacts shows a $1 / d$ dependence. Measured at $4.2 \mathrm{~K}$. 
tions even at high temperature due to the nanoscopic size of the contact. We have also demonstrated that phonon spectroscopy in atomic contacts is possible opening the possibility of studying inelastic processes in an atomic scale.
We acknowledge fruitful discussions with J.J. Sáenz, J. van Ruitenbeek, C. Urbina, A. Levy Yeyati, and A. MartínRodero. This work has been supported by the spanish DGICyT under Contract No. PB97-0068.
*Present address: GM/VSM, Postbus 9504, 2300 RA Leiden, The Netherlands. Email: untiedt@phys.leidenuniv.nl

${ }^{1}$ N. Agraï, J. G. Rodrigo, and S. Vieira, Phys. Rev. B 47, 12345 (1993).

${ }^{2}$ C. J. Muller, J. M. van Ruitenbeek, and L. J. de Jongh, Physica C 191, 485 (1992).

${ }^{3}$ J. A. Torres, J. I. Pascual, and J. J. Sáenz, Phys. Rev. B 49, 16 581 (1994).

${ }^{4}$ M. Brandbyge, M. R. Sörensen, and K. W. Jacobsen, Phys. Rev. B 56, 14956 (1997).

${ }^{5}$ A. M. Bratkovsky, A. P. Sutton, and T. N. Todorov, Phys. Rev. B 52, 5036 (1995).

${ }^{6}$ J. Ferrer, A. Martín-Rodero, and F. Flores, Phys. Rev. B 38, 10 113 (1988).

${ }^{7}$ R. Landauer, Philos. Mag. 21, 863 (1970).

${ }^{8}$ E. Scheer, N. Agraï, J. C. Cuevas, A. Levy Yeyati, B. Ludolph, A. Martín-Rodero, G. Rubio Bollinger, J. M. van Ruitenbeek, and C. Urbina, Nature (London) 394, 154 (1998); see also E. Scheer, P. Joyez, D. Esteve, C. Urbina, and M. H. Devoret, Phys. Rev. Lett. 78, 3535 (1997).

${ }^{9}$ J. C. Cuevas, A. Levy Yeyati, A. Martín-Rodero, G. Rubio Bollinger, C. Untiedt, and N. Agrait, Phys. Rev. Lett. 81, 2990 (1998); same behavior in larger contacts Daniel Sanchez-Portal, Carlos Untiedt, Jose M. Soler, Juan J. Sáenz, and N. Agraï, ibid. 79, 4198 (1997).

${ }^{10}$ G. Rubio, N. Agrait, and S. Vieira, Phys. Rev. Lett. 76, 2302 (1996); same behavior in larger contacts N. Agraït, G. Rubio, and S. Vieira, ibid. 74, 3995 (1995).

${ }^{11}$ B. Ludoph, M. H. Devoret, D. Esteve, C. Urbina, and J. M. van Ruitenbeek, Phys. Rev. Lett. 82, 1530 (1999); see also D. L. Maslov, C. Barnes, and G. Kirczenow, ibid. 70, 1984 (1993).

${ }^{12}$ P. A. Lee and A. D. Stone, Phys. Rev. Lett. 55, 1622 (1985); P. A. Lee, A. D. Stone, and H. Fukuyama, Phys. Rev. B 35, 1039 (1987).

${ }^{13}$ A. van Oudernaarden, M. H. Devoret, E. H. Visscher, Yu. V. Nazarov, and J. E. Mooij, Phys. Rev. Lett. 78, 3539 (1997).

${ }^{14}$ P. A. M. Holweg, J. A. Kokkedee, J. Caro, A. H. Verbruggen, S. Radelaar, A. G. M. Jansen, and P. Wyder, Phys. Rev. Lett. 67, 2549 (1991).

${ }^{15}$ Randall M. Feenstra, Surf. Sci. 299/300, 965 (1994).

${ }^{16}$ Y. Hasegawa and Ph. Avouris, Phys. Rev. Lett. 71, 1071 (1993); M. F. Crommie, C. P. Lutz, and D. M. Eigler, Nature (London) 524, 363 (1993).

${ }^{17}$ M. F. Crommie, C. P. Lutz, and D. M. Eigler, Science 262, 218 (1993).

${ }^{18}$ In the sample used in our experiments the measured residual resistivity ratio (RRR) is 29 , which implies a mean free path $l$ of about $1 \mu \mathrm{m}$. Since $l=1 / n \sigma_{c}$, where $\sigma_{c}$ is the scattering cross section of the impurities, this is consistent with the nominal impurity concentration of our samples $(100 \mathrm{ppm})$ for which the mean distance between impurities is about $55 \AA$.

${ }^{19}$ I. K. Yanson Zh. Éksp. Teor. Fiz. 66, 1035 (1974) [Sov. Phys. JETP 39, 1069 (1974)]; See also A. G. M. Jansen, A. P. van Gelder, and P. Wyder, J. Phys. C 13, 6073 (1980). 